\title{
Physical gelation of crystallizing metallocene and Ziegler-Natta ethylene-hexene copolymers
}

\author{
Mikhail Gelfer ${ }^{1}$, Roland H. Horst, H. Henning Winter*, Amy M. Heintz, Shaw Ling Hsu \\ Department of Polymer Science and Engineering and Department of Chemical Engineering, University of Massachusetts, Amherst, MA 01003, USA
}

Received 17 September 2002; received in revised form 13 January 2003; accepted 15 January 2003

\begin{abstract}
The effect of molecular architecture on the evolution of viscoelastic properties during crystallization was investigated using ethylenehexene copolymers manufactured via metallocene (M-LLDPE) and Ziegler-Natta (ZN-LLDPE) processes. Differences in branching distribution were shown to have a drastic effect on the viscoelastic properties near the gel point. It is shown that the branching distribution rather than branch content is the determining parameter for the evolution of the rheological properties during isothermal and non-isothermal crystallization, and for the width of the solidification interval. We developed a partial melting technique for the preparation of stable critical gels of LLDPE whose viscoelastic properties correspond to the intermediate state between melt and solid. Local molecular conformation and crystallinity in these gels were characterized by Raman spectroscopy, which shows that the transition from melt-like to solid-like rheological behavior (physical gelation) in LLDPE occurs at a very low overall crystallinity of less than $5 \%$.

(C) 2003 Elsevier Science Ltd. All rights reserved.
\end{abstract}

Keywords: Gelation; Crystallization kinetics; Polyethylene

\section{Introduction}

The development of the metallocene process allows synthesizing ethylene- $\alpha$-olefin copolymers (LLDPEs) inaccessible by traditional Ziegler-Natta synthesis. Significant structural differences exist between novel and conventional (Ziegler-Natta) copolymers. Intramolecular branching distributions in both metallocene and conventional ethylene- $\alpha$ olefin copolymers were shown to be quite similar: branched units are more isolated than it is predicted by Bernoullian statistics. This deviation is insignificant when the content of branched units $x$ is low $(x<2$ mol\%), however, it increases for more branched polymers $(x>10 \mathrm{~mol} \%)$ [1-4]. On the other hand, intermolecular composition distributions in these systems are drastically different [5-7]. ZN-LLDPEs are highly heterogeneous copolymers with significant contents of highly and low-branched molecules [8]. In contrast, in metallocene systems branched units are statistically distributed among polymer chains [5-7]. It is

\footnotetext{
* Corresponding author. Tel.: + 1-413-545-0922; fax: + 1-413-545-1647. E-mail address: winter@ecs.umass.edu (H.H. Winter).

1 Present address. State University of New York at Stony Brook, Department of Chemistry, Stony Brook, NY 11794, USA.
}

well known that melting temperatures and equilibrium crystallinity in LLDPEs may be strongly influenced by the branching distribution [9-13].

During crystallization, $\alpha$-olefin segments accumulate in the amorphous region since the branches of two carbons or higher are excluded from the crystals. Because of this changing melt composition the melting point does not directly reflect the crystal thickness [14]. Metallocene ethylene- $\alpha$-olefin copolymers show a very large temperature range of melting/crystallization [15] due to the broad distribution of crystallizable sequence lengths. In isothermal crystallization, the crystallinity initially increases rapidly followed by a very slow increase at longer times [16]. Ziegler-Natta copolymers, on the other side, exhibit a bimodal (intermolecular) distribution of crystallizable sequence lengths with a substantial fraction of longer crystallizable sequences that crystallize rapidly at comparatively high temperatures. ZN-LLDPE also contains a considerable fraction of highly branched chains with a high concentration of very short crystallizable sequences. In this work, experiments were performed at relatively high temperatures $\left(T>110^{\circ} \mathrm{C}\right)$, significantly exceeding $T_{\mathrm{g}}$ of LLDPEs. At these temperatures, the contribution of shortest linear segments into crystallization is insignificant. 
Rheology during isothermal crystallization was found to be pronouncedly influenced by such differences in molecular architecture [17] while melt rheology at elevated temperatures was comparatively insensitive.

This study explores the influence of branching distribution on viscoelastic behavior of LLDPEs during nonisothermal crystallization and melting. Time resolved mechanical spectroscopy during a cooling scan shows the effect of branch distribution on the width of the solidification interval. Using a previously developed method [18], we prepared stable critical physical gels, whose viscoelastic properties correspond to the intermediate state between melt and solid, with the purpose of studying their properties. In this work we developed a partial melting technique for the preparation of stable critical gels from LLDPEs, whose overall branching does not exceed 4 mol\%. Local molecular conformation and crystallinity near the gel point were characterized by Raman spectroscopy.

\section{Physical gelation by crystallization}

When a polymer crystallizes from the melt state it undergoes a liquid to solid transition. At the transition the polymer has properties neither of a liquid nor of a solid. A sample at this transition point is called a critical gel. Just beyond this transition, the sample is an extremely soft solid that readily deforms as soon as any stress is applied.

This transient state during solidification may arise from different morphologies: (1) polymers forming large and well-defined spherulitic structures, or (2) polymers forming very small spherulites or no spherulites at all. A typical example for case (1) is isotactic polypropylene [19,20]. At high enough temperatures it exhibits a very low nucleation density and, therefore, large spherulites can grow. Percolation results in a yield effect, the system behaves similar to a suspension of solid particles (spherulites) in a liquid matrix (amorphous melt). Solidification occurs when the volume fraction of the spherulites becomes large enough. The crystallinity of the spherulites is still low at percolation conditions, so that the overall crystallinity at the gel point is small.

Case 2 results from high nucleation densities and/or high branching contents. $\alpha$-olefin ethylene copolymers as used in this study are a typical example. These systems crystallize at lower temperatures resulting in a higher nucleation density. They form very small crystalline entities. Because of the small size of the solid particles these systems are best described as suspension of minute particles or even as physical networks when the size of the crystallites is particularly small at the gel point. Indeed, it is known [21] that the same polymer chain can go through several amorphous and crystalline regions. Thus crystalline regions can be presented as cross-links, linking together several neighboring chains. This mechanism of physical crosslinking is analogous to the network formation in chemical gelation. Highly branched ethylene-butene copolymers [18] show a crystallinity at the gel point of about $0.5 \% \mathrm{w} / \mathrm{w}$. In the present work, we will characterize critical gels using in situ Raman spectroscopy measurements.

Drawing conclusions about the crystallization from the rheological properties is not possible without taking these morphological parameters into account. The most prominent are nucleation density and size of the crystal entities. Rheological data can only be compared within groups of semi-crystalline polymers that exhibit similar morphology. An indication for these basic differences is the degree of crystallinity at the gel point, which is considerably higher for case 1 (app. 5\%) than for case 2 (app. 0.5\%).

It was shown experimentally that the rheological pattern of gelation [22-25] adequately describes liquid-solid transitions in flexible-chain polymers induced by crystallization or melting. The melt-solid transition during crystallization manifests itself by the slow power-law dynamics at low frequencies. Experimentally, this shows as 'plateau' in the loss tangent curve, $\tan \delta(\omega)[26,27]$. The evolution of the rheological properties of crystallizing polymers is consistent with that of physical the gelation processes.

\section{Experimental}

\subsection{Materials}

Branching content and molar mass parameters of the samples under investigation are listed in Table 1. The ethylene-hexene copolymers M-LLDPE and ZN-LLDPE were manufactured by ExxonMobil and were selected to represent the different types of composition distributions, occurring in industrial ethylene $\alpha$-olefin systems. Indeed, the composition distribution for M-LLDPE is typical for metallocene LLDPEs, while the composition distribution of ZN-LLDPE is representative of Ziegler-Natta systems [17]. M-LLDPE and ZN-LLDPE were characterized by temperature raising elution fractionation (TREF) at ExxonMobil. According to TREF data [17], the intermolecular branching distribution $P(x)$ in ZN-LLDPE is bimodal, with relatively high fractions of both low-branched chains (branching content $x<1 \mathrm{~mol} \%)$ and highly branched chains $(5<x<$ $12 \mathrm{~mol} \%$ ). This differs from the broad unimodal branching distribution in M-LLDPE, which can be described as skewed Gaussian centered at $x_{\text {avg }}=2.6 \mathrm{~mol}$. In the metallocene copolymer the contents of highly $(x>5 \times$ mol\%) and low-branched $(x<1 \mathrm{~mol} \%)$ chains are low.

Ziegler-Natta HDPE (ZN-HDPE), commercially manufactured by ExxonMobil and metallocene HDPE (MHDPE) custom-manufactured by Exxon served as reference materials in order to elucidate the effect of branching and molecular weight distribution on rheology and crystallization behavior of ethylene copolymers. LLDPE/HDPE 
Table 1

Characteristics of the investigated ethylene $\alpha$-olefin copolymers

\begin{tabular}{|c|c|c|c|c|c|}
\hline Polymer & Method of synthesis catalyst & Content of branched units 1-hexene (mol\%) & Density $\left(\mathrm{g} / \mathrm{cm}^{3}\right)$ & $M_{\mathrm{w}}(\mathrm{kg} / \mathrm{mol})^{\mathrm{a}}$ & $M_{\mathrm{w}} / M_{\mathrm{n}}^{\mathrm{a}}$ \\
\hline ZN-LLDPE & Ziegler-Natta & 3.8 & 0.917 & 115 & 4.0 \\
\hline M-LLDPE & Metallocene & 2.61 & 0.918 & 107 & 2.4 \\
\hline ZN-HDPE & Ziegler-Natta & 0 & 0.965 & $\sim 80$ & $\sim 5$ \\
\hline M-HDPE & Metallocene & 0 & 0.97 & 110 & $\sim 3$ \\
\hline
\end{tabular}

${ }^{\text {a }}$ Determined by GPC at EXXON, Baytown, TX.

blends were prepared by melt-blending M-LLDPE and ZHD at $180^{\circ} \mathrm{C}$ in a Brabender extruder.

Thermal analysis experiments were performed under nitrogen atmosphere in a Perkin-Elmer calorimeter DSC 7 with water-bath cooling. The instrument was calibrated with high-purity standards of indium and tin. The sample was kept at a preheating temperature of $160{ }^{\circ} \mathrm{C}$ for a $10 \mathrm{~min}$ to remove influences of thermal history as much as possible; then it was quenched at $320 \mathrm{~K} / \mathrm{min}$ to a given crystallization temperature and kept there for some time. In a subsequent DSC heating scan $(10 \mathrm{~K} / \mathrm{min})$, the heat of fusion, $\Delta H_{\mathrm{m}}$, was determined. Dividing by the heat of fusion for the polyethylene crystal gives the crystallinity, $\phi$.

Oscillatory shear measurements were performed on the strain-controlled rheometer ARES LS 3 (Rheometric Scientific). All rheological experiments were done with $25 \mathrm{~mm}$ parallel plates. The temperature scale in the rheological measurements was calibrated to the melting point of benzophenone and benzoic acid standards. The frequency range $0.1-100 \mathrm{rad} / \mathrm{s}$ of the dynamic experiments covered sufficiently low frequencies allowing for the observation of the characteristic rheological patterns of critical gels. The measuring time for single data points was short enough, so that the rheological properties of crystallizing samples did not undergo significant change during individual measurements. Small strain amplitudes, $\gamma_{\mathrm{a}}=$ 0.01-0.03, were chosen in order to ensure minimal influence of imposed strain on the crystallization process. At these small values the gel time does not show a dependence on the strain amplitude. Therefore, the crystallization should not be influenced as well.

Rheological experiments were conducted according to three thermal protocols: isothermal and non-isothermal crystallization, and stepwise melting. All crystallization experiments started out by initially holding the samples at $160{ }^{\circ} \mathrm{C}$ for $10 \mathrm{~min}$ with the purpose of erasing the effect of prior thermal history. For the isothermal crystallization, samples were rapidly cooled to the experimental temperature and held there while monitoring the growth of the dynamic moduli. Non-isothermal rheological data were acquired in a cooling ramp that started at $160{ }^{\circ} \mathrm{C}$. A low cooling rate of $4 \mathrm{~K} / \mathrm{h}$ was slow enough to allow for data acquisition at low frequencies. The stepwise melting temperature protocol was specifically designed for the preparation of stable physical gels from LLDPEs. The sample was first cooled $(1 \mathrm{~K} / \mathrm{min})$ to $25^{\circ} \mathrm{C}$ for crystal- lization, then slowly heated to the temperature of the first isothermal step $T_{1}$ and held there isothermally for $300 \mathrm{~min}$. The temperature was then increased at small temperature increments of $\Delta T=T_{i+1}-T_{i}=1 \mathrm{~K}$. The annealing process $\left(300 \mathrm{~min}\right.$ ) was repeated at each temperature $T_{\mathrm{i}}$ before the experiments were carried out.

\subsection{FT Raman measurements}

Fourier transform Raman spectra were recorded on a Bruker FRA 106 spectrometer equipped with a Nd/YAG laser $(1.064 \mu \mathrm{m})$. The excitation/collection geometry was $180^{\circ}$ and spectral resolution was maintained at $4 \mathrm{~cm}^{-1}$. Laser power at the sample was $200 \mathrm{~mW}$, and 1024 scans were signal averaged. The temperature program used in the Raman experiments duplicates those described above for partial melting and was carried out inside the spectrometer under $\mathrm{N}_{2}$. The sample was packed into a glass capillary and heated by a cell attached to an Omega temperature controller. The temperature was calibrated to the melting point of benzoic acid and was stable to $\pm 0.5 \mathrm{~K}$. Heating due to the laser was negligible at temperatures above $50{ }^{\circ} \mathrm{C}$.

Band deconvolution procedures were employed to calculate the degree of crystallinity using the Bruker Opus software package. A baseline was drawn from 1600 to $960 \mathrm{~cm}^{-1}$ and then the spectra were normalized to the total integrated intensity of the $\mathrm{CH}_{2}$ twisting region (1340$1241 \mathrm{~cm}^{-1}$ ). Band widths and shapes were fixed according to the literature [28]. The Levenberg-Marquardt algorithm was used to fit the intensities and positions.

To ensure compatibility of temperature scale in Raman and rheological experiments, all temperature controllers were calibrated to the melting point of the benzoic acid standard.

\section{Results and discussion}

\subsection{Melt properties}

Dynamic and steady-shear rheological properties of LLDPEs and HDPEs above the melting temperature are presented in Fig. 1. For all samples under investigation, the slopes of the storage modulus, $G^{\prime}$, and the loss tangent, $\tan \delta$, as a function of angular frequency, $\omega$, do not reach the low frequency values of 2 and 1 within the experimentally 

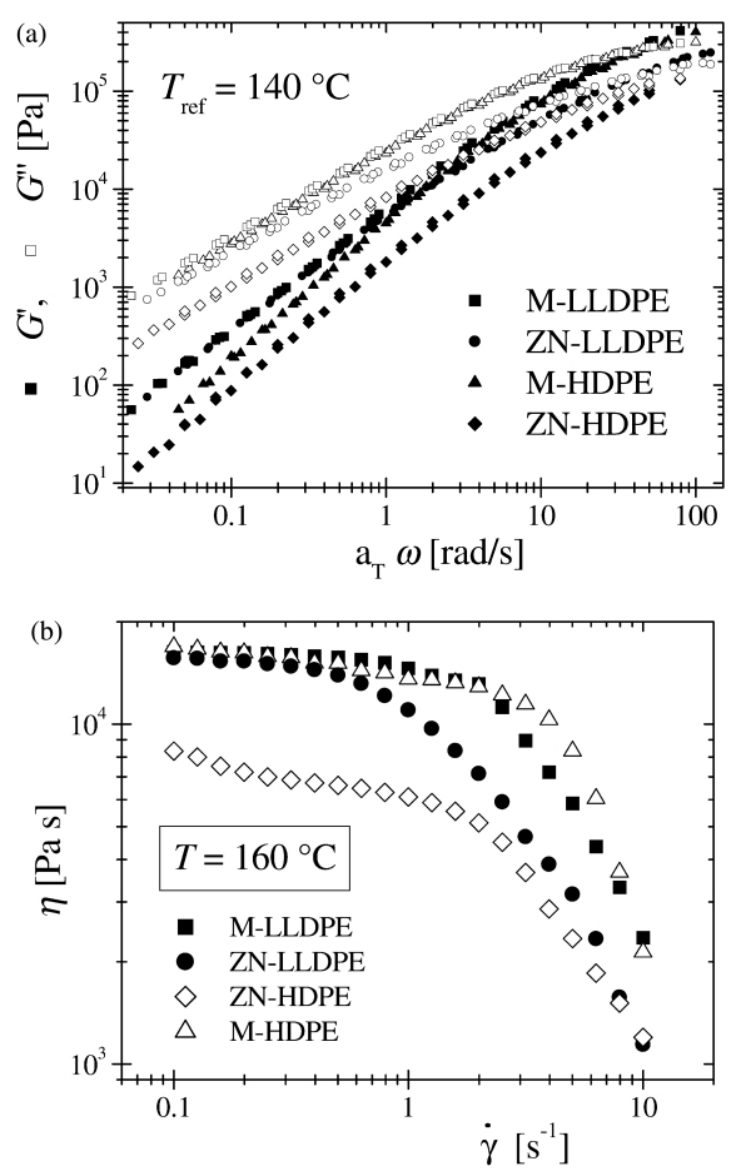

Fig. 1. (a) Frequency dependence of the dynamic moduli for ethylene copolymers. $T_{\text {ref }}=140{ }^{\circ} \mathrm{C} ; a_{\mathrm{T}}$ is the logarithmic shift factor. (b) Shear rate dependence of the steady shear viscosity for ethylene copolymers, $T=160^{\circ} \mathrm{C}$

accessible frequency range. ZN-HDPE was only available to us with a slightly lower average molecular weight compared to the other polymers under investigation. This affords lower values of $G^{\prime}$ and steady shear viscosity, $\eta$.

The flow activation energy, $E_{\alpha}$, is slightly lower in the Ziegler-Natta polymers than in the metallocene systems (Table 2). In steady shear rate experiments, the onset of the power-law behavior occurred at lower shear rates in ZieglerNatta systems than in metallocene analogs. The experiments also show that in the tested systems, the effect of branching distributions on melt rheology is insignificant. A lower flow activation energy, the earlier onset of shear thinning, and the

Table 2

Characteristics of the investigated ethylene $\alpha$-olefin copolymers

\begin{tabular}{lll}
\hline Polymer & $\begin{array}{l}\text { Flow activation energy, } E_{\alpha} \\
(\mathrm{kJ} / \mathrm{mol})\end{array}$ & $\begin{array}{l}\left(\frac{\partial \ln G^{\prime}}{\partial \ln \omega}\right) \\
(0.01 \mathrm{rad} / \mathrm{s}<\omega<1 \mathrm{rad} / \mathrm{s})\end{array}$ \\
\hline ZN-LLDPE & 25 & 1.12 \\
M-LLDPE & 27 & 1.30 \\
ZN-HDPE & 23 & 1.16 \\
M-HDPE & 24 & 1.15 \\
\hline
\end{tabular}

weaker dependence of viscoelastic functions on frequency observed in ZN-LLDPE all indicate a broader molecular weight distribution for the ZN-LLDPE [29,30].

\subsection{Isothermal crystallization}

Crystallization kinetics was determined by DSC inferred from heats of fusion after different crystallization times. The growing crystallinity, $\phi(t)$, during isothermal crystallization of ZN-LLDPE and M-LLDPE is presented in Fig. 2. In spite of higher overall branching, the ZN-LLDPE sample crystallizes faster and at higher temperatures than MLLDPE. The rate of the isothermal crystallization is significantly higher for low-branched chain segments [31, 32], thus higher bulk crystallization rates in ZN-LLDPE are attributed to the significant content in that copolymer of low-branched molecules whose crystallization rate is higher than in more branched analogs [31]. Crystallites formed by these almost linear molecules have a higher melting temperature. According to literature data [33], such crystallites form the backbone of supermolecular structures (spherulites). A significant fraction of the crystallinity
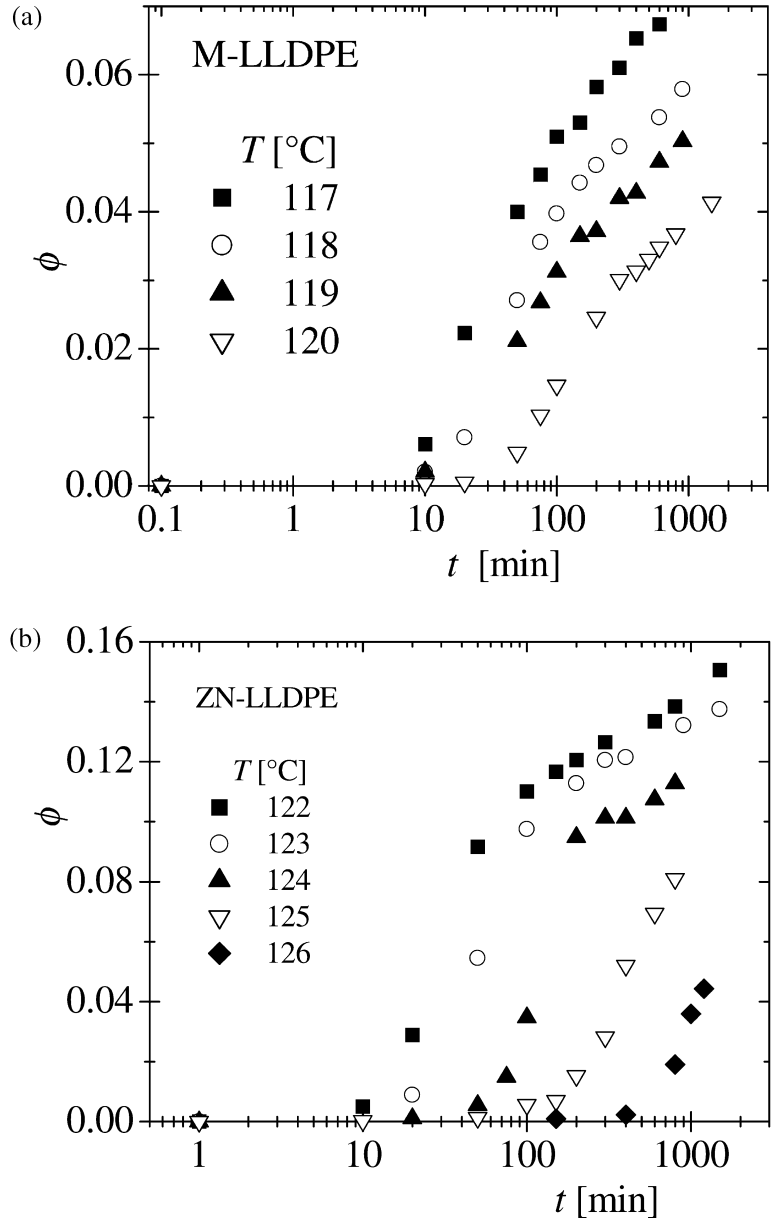

Fig. 2. Crystallinity during the isothermal crystallization of (a) M-LLDPE and (b) ZN-LLDPE. 
develops only after the spherulites have become volume filling.

The gel time $\left(t_{\text {gel }}\right)$ was determined rheologically by the first appearance of a flat $\tan \delta(\omega)$ curve at low frequencies as described in Ref. [18]. The corresponding crystallinity, $\phi_{\mathrm{gel}}=\phi\left(t_{\mathrm{gel}}\right)$, was read from the DSC curves $\phi(t)$. Fig. 3 shows the dependence of $G^{\prime}$ and $\tan \delta$ on the crystallinity for an angular frequency of $\omega=1 \mathrm{rad} / \mathrm{s}$. At the gel point, the slope of $G^{\prime}(\phi)$ and of $\tan \delta(\phi)$ changes. The shapes of $G^{\prime}(\phi)$ and $\tan \delta(\phi)$ curves are distinctly different for M-LLDPE and ZN-LLDPE. This indicates structural differences in the corresponding physical gels. It is known that the presence of branched units hinders formation of well-defined spherulites [5,34]. Indeed, branched molecules require lower temperatures to crystallize, as compared to linear analogs, so larger number of nuclei exists for the same overall crystallinity drastically decreasing the average spherulite size. In addition, the presence of short branches slows down the growth of crystallites and hinders formation of perfect crystals forming the backbone of well-defined supermolecular structures. As a result the almost linear chains present in ZN-LLDPE form larger spherulitic structures visible in micrographs. Nevertheless, even in ZN-LLDPE the branching content is too high to allow the growth of large HDPElike spherulites. In contrast, all chains of M-LLDPE contain
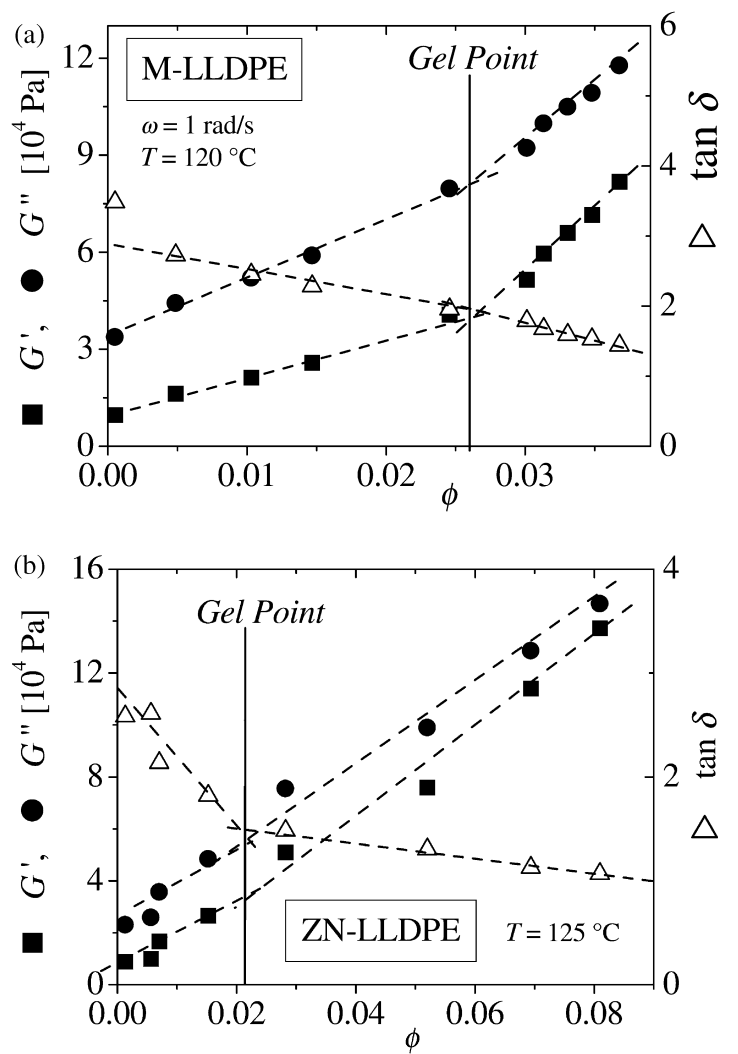

Fig. 3. Crystallinity dependence of viscoelastic properties near the gel point during the isothermal crystallization ( $\omega=1 \mathrm{rad} / \mathrm{s}$ ); (a) M-LLDPE- $120{ }^{\circ} \mathrm{C}$; (b) ZN-LLDPE- $125^{\circ} \mathrm{C}$. The gel point has been determined by the occurrence of a plateau in $\tan \delta(\omega)$. branched units, so that the crystalline entities will remain small and not well defined.

\subsubsection{Non-isothermal crystallization (cooling ramp)}

The evolution of viscoelastic properties of M-LLDPE, ZN-LLDPE and ZN-HDPE during slow cooling $(4 \mathrm{~K} / \mathrm{h})$ is presented in Fig. 4. It can be seen that the melt-solid transition due to crystallization results in a steep increase in $G^{\prime}$ and a simultaneous drop in $\tan \delta$. While this transition also results in a change from the melt-like to solid-like rheological behavior according to Winter's criteria (i.e. positive slope at $\tan \delta(\omega)$ dependencies beyond the gel point) the rapid crystallization at lower temperatures does
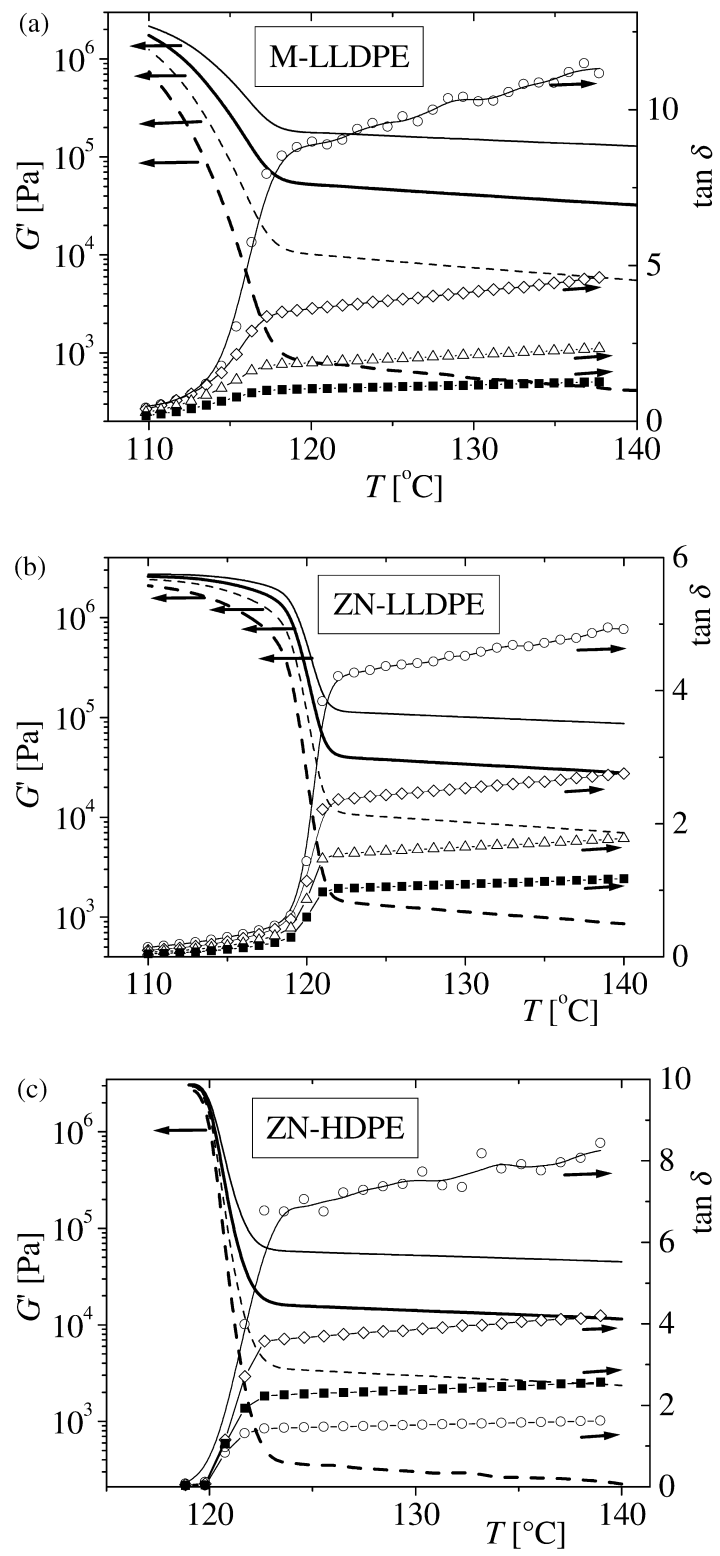

Fig. 4. Evolution of dynamic viscoelastic properties of LLDPEs during nonisothermal crystallization (cooling ramp $4 \mathrm{~K} / \mathrm{h}$ ); (a) M-LLDPE; (b) $\mathrm{ZN}$ LLDPE; (c) ZN-HDPE. Lines: storage modulus $G^{\prime}$, symbols: loss tangent, $\tan \delta ; \omega=0.2 \mathrm{rad} / \mathrm{s}$ (thick dashed line, circles), 1.26 (thin dashed, diamonds), 5 (thick solid, triangles), and $20 \mathrm{rad} / \mathrm{s}$ (thin solid, full squares). 
not allow for an efficient acquisition of rheological data in the low-frequency region. Instead we suggest that for a nonisothermal transition the slope of $G^{\prime}(T)$ near the melt-solid transition may serve as the direct measure of the widths of 'solidification interval' defining transition between meltlike and solid-like mechanical behavior, the larger slope corresponding to the narrower transition interval. The slope of $G^{\prime}(T)$ is directly related to the system's mechanical properties and is a much more reliable measure of the solidification behavior than the widths of DSC peaks acquired during crystallization. There is no simple relation between crystallinity and rheological properties as discussed above. Indeed, the properties of the transient network formed during crystallization may be influenced by the character of supermolecular structures, which in turn are affected by cooling rate $[5,13]$. As a result the same overall DSC crystallinity may correspond to different mechanical properties, so drawing conclusion from DSC exotherms on a system's mechanical behavior can only be done accounting for morphological information. In addition, the widths of a DSC peaks may be strongly affected by re-crystallization and co-crystallization processes whose relationship to the viscoelastic behavior of a crystallizing polymer is even more difficult to determine.

It may be seen that the liquid-solid transition during non-isothermal crystallization of M-LLDPE occurs at lower temperatures and over a broader temperature range than in the Ziegler-Natta system (Fig. 4(a) and (b)). The steepest transition at highest temperature was observed in linear polymer ZN-HDPE (Fig. 4(c)). The solidification interval as determined by rheology agrees well with the findings for the crystallization range that is much broader for the random, metallocene catalyzed copolymers [15].

The results strongly suggest that the presence of a sufficient fraction of molecules with low content of branched units $(x<0.75 \mathrm{~mol} \%)$ determines the solidification behavior during non-isothermal crystallization of ethylene copolymers. In order to test this hypothesis, we investigated the rheological behavior of ZN-HDPE/MLLDPE blends during non-isothermal crystallization

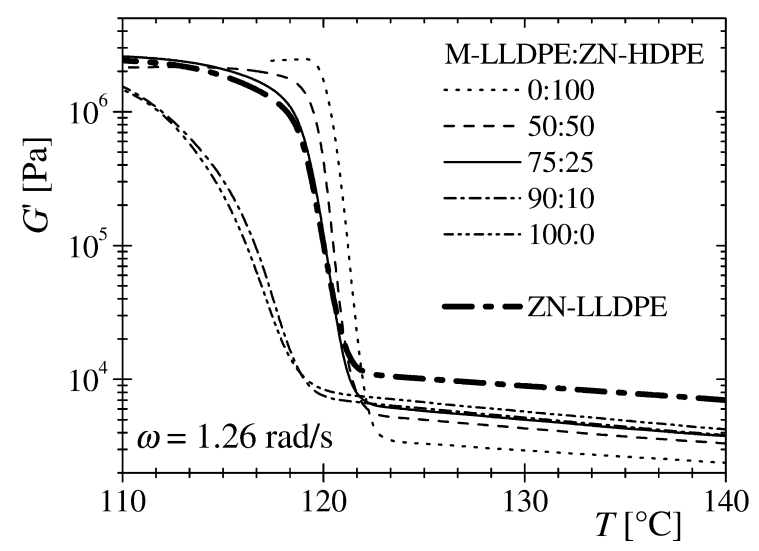

Fig. 5. Evolution of storage modulus $G^{\prime}(T)$ during slow cooling $(4 \mathrm{~K} / \mathrm{h})$ of M-LLDPE/ZN-HDPE blends.
Table 3

Slope of the logarithm of the storage modulus with respect to temperature at the transition for M-LLDPE/ZN-HDPE blends as shown in Fig. 5 at an angular frequency of $\omega=1.26 \mathrm{rad} / \mathrm{s}$

\begin{tabular}{ll}
\hline Blend composition $(\mathrm{w} / \mathrm{w} \%)$ & Slope at transition $\left(\partial \ln G^{\prime} / \partial T\right)\left(\mathrm{K}^{-1}\right)$ \\
\hline 0 (ZN-HDPE) & 1.35 \\
50 & 0.91 \\
75 & 0.85 \\
90 & 0.36 \\
100 (M-LLDPE) & 0.34 \\
ZN-LLDPE & 0.84 \\
\hline
\end{tabular}

(Fig. 5 and Table 3). It can be seen that while the increase in ZN-HDPE content in the blend from 10 to 50 [w/w\%] enhances overall composition heterogeneity it also causes the narrowing of the solidification interval, i.e. the temperature range where the rheological properties change from that of a liquid to that of a solid. For instance, the solidification interval for ZN-LLDPE is as narrow as for the ZN-HDPE/M-LLDPE blend containing 25 [w/w\%] of ZNHDPE. The overall branching is almost twice as high in $\mathrm{ZN}$ LLDPE as in the blend (3.8 vs. $1.95 \mathrm{~mol} \%$ ). However, the content of low-branched chains $(0<x<0.75 \mathrm{~mol} \%)$ is quite similar in these systems ( 24 vs. $25 \mathrm{~mol} \%$ ). These observations indicate that the rheological behavior in the vicinity of liquid-solid transition is indeed determined by the content of linear or almost linear molecules, rather than by the overall branching.

\subsubsection{Stable critical gels by partial melting}

The overall crystallinity and conformation of molecular chains in the vicinity of a gel point are of particular interest. However, physical gels formed during crystallization are transient structures whose structure and properties change as crystallization proceeds. Thus it may be difficult to obtain the material which will stay near the gel point for an amount of time sufficient for the application of such structural methods as solid-state NMR or FTIR and steady-shear rheological techniques usually applied for the characterization of physical gels [35]. The low density contrast between crystalline and molten LLDPEs also hinders application of real-time SAXS and SALS techniques when the sample's crystallinity is low.

In this work, we developed the partial melting temperature protocol which allows decreasing the LLDPE crystallinity in controlled increments, making it possible to approach the gel point and stay near it for a period of time sufficient for the structural characterization. The proposed method is based on a well-known correlation between branching and melting temperature in LLDPEs. Indeed, exclusion of branched units from the crystalline domain limits the maximal lamellar thickness in crystallites formed by ethylene- $\alpha$-olefin copolymers $[14,32,34]$. Because of the slow crystallization kinetics of these copolymers, it is much faster to achieve an equilibrated material with exactly the 
necessary amount of crystallinity to reach the gel point by partial melting [36]. Annealing the material at high temperatures causes melting of the less stable crystals, but at the same time partial recrystallization and perfection of the crystals. This leads to a pronounced increase in melting temperature [18].

Obviously, the stepwise partial melting procedure allows for the preparation of stable critical gels, whose crystallinity $\phi$ remains in the vicinity of critical gel separating melt-like and solid-like behavior for a period of time, sufficient for the structural characterization [36]. Furthermore, the partial melting program includes only heating steps followed by prolonged equilibration so that chains in low melting crystals can mobilize and recrystallize. The stepwise melting and recrystallization procedures can be reliably implemented in the environmental chamber of the rheometer and measuring cell of the spectroscopic device.

The occurrence of a solid-melt transition during partial melting was detected by monitoring of viscoelastic properties by oscillatory shear. Indeed, a flat $\tan \delta(\omega)$ dependence at low frequencies, indicative of a near-critical state was observed for physical gels, prepared by partial melting of M-LLDPE and ZN-LLDPE (Fig. 6). It can be seen that critical gels may be prepared both from metallocene and from Ziegler-Natta LLDPEs.

Raman spectra, recorded during partial melting of $\mathrm{M}-$ LLDPE are presented in Fig. 7 Information regarding the chain packing and chain conformation can be derived from
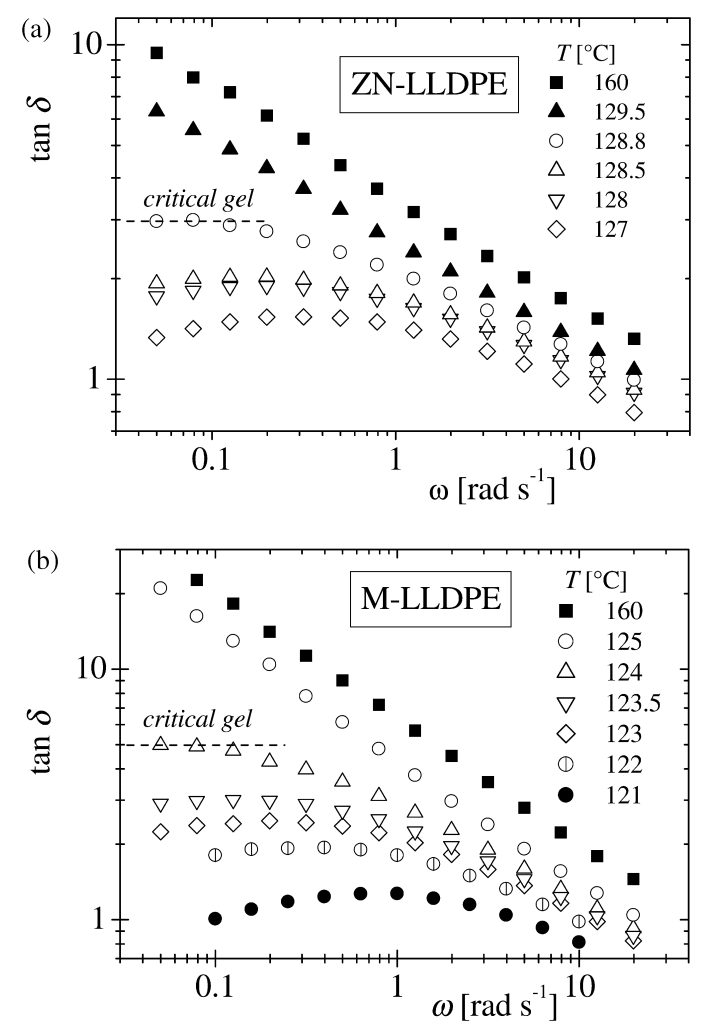

Fig. 6. Viscoelastic properties of stable physical gels formed during partial melting of LLDPEs. (a) ZN-LLDPE (b) M-LLDPE.

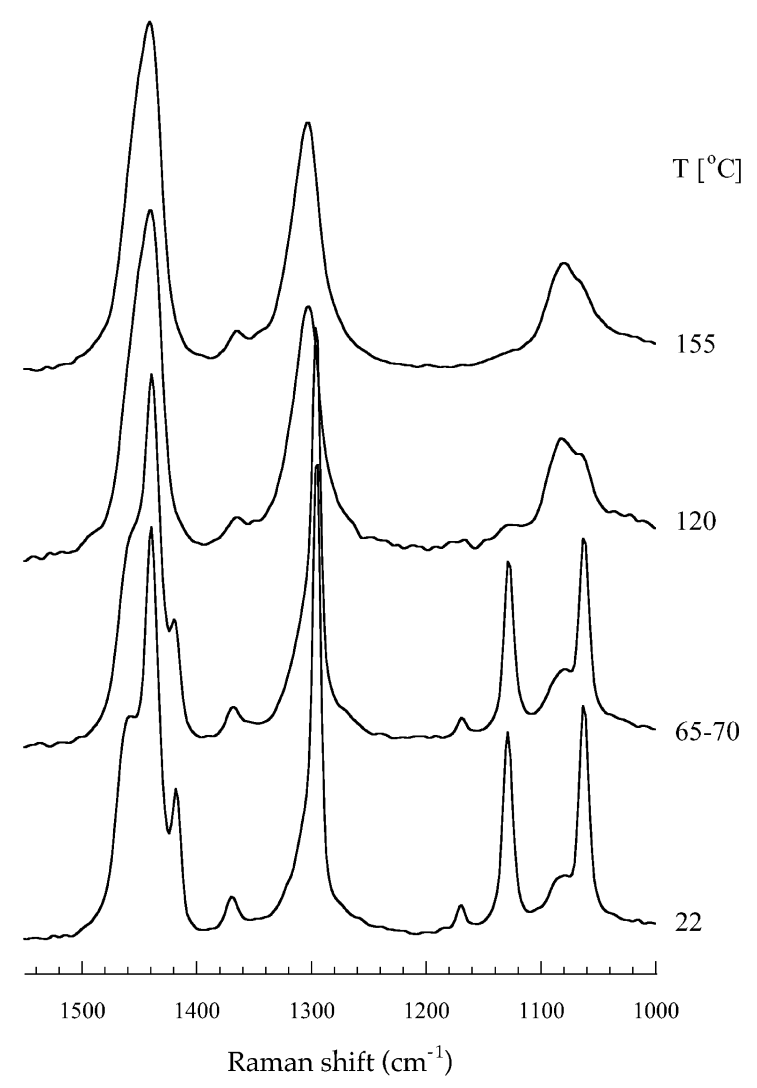

Fig. 7. Raman spectra, acquired during partial melting of M-LLDPE.

the Raman spectral region from 1600 to $150 \mathrm{~cm}^{-1}$. In particular, the band at $1416 \mathrm{~cm}^{-1}$ arises from orthorhombic crystal field splitting, and those at 1060 and $1130 \mathrm{~cm}^{-1}$ are characteristic of long trans sequences. Both regions have been used to determine the degree of crystallinity in polyethylene samples [37-41]. Strobl et al. [37,38] calculated the degree of crystallinity by dividing the intensity of the $1416 \mathrm{~cm}^{-1}$ band by the total $\mathrm{CH}_{2}$ twisting intensity and comparing with the value obtained for a completely crystalline sample. Naylor et al. [41] instead, used the ratio of the $1060 \mathrm{~cm}^{-1}$ skeletal stretch to the $\mathrm{CH}_{2}$ twist. The two methods are in good agreement for calculating the degree of crystallinity of HDPE. In contrast, for LLDPE, crystallinity based on the intensity at $1416 \mathrm{~cm}^{-1}$ is usually below the one derived from the $1060 \mathrm{~cm}^{-1}$ band [41].

Both methods are used here to estimate the crystallinity. Examples of the band deconvolution of the $\mathrm{CH}_{2}$ bending and skeletal stretching regions are shown in Fig. 8(a) and (b), respectively. Using the areas found for the 1416 and the $1060 \mathrm{~cm}^{-1}$ regions, the temperature dependence of crystallinity can be calculated and is shown in Fig. 9. The deconvoluted band areas are shown in Table 4.

At room temperature, the difference in crystallinity calculated from the vibrations at 1416 vs. $1060 \mathrm{~cm}^{-1}$ is quite large, approximately $12 \%$. As the temperature is increased, this difference decreases to $5 \%$ at $120{ }^{\circ} \mathrm{C}$. Above this temperature, the band at $1416 \mathrm{~cm}^{-1}$ disappears, 

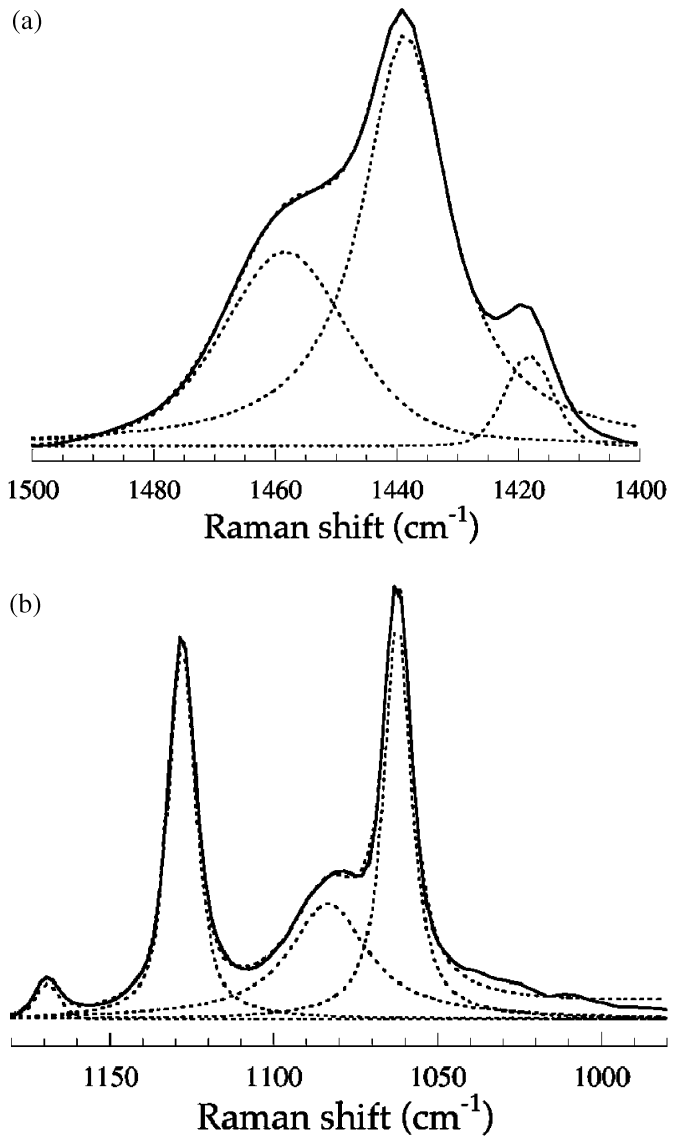

Fig. 8. Example of a band deconvolution of the Raman spectra; (a) $\mathrm{CH}_{2}$ bending region, where the $1416 \mathrm{~cm}^{-1}$ band is characteristic of orthorhombic crystals; (b) skeletal stretching region, where the $1060 \mathrm{~cm}^{-1}$ band is characteristic of all trans sequences.

indicating the absence of orthorhombic crystal field splitting. Yet the crystallinity at the gel point, $123.5^{\circ} \mathrm{C}$, determined from the overall intensity at $1060 \mathrm{~cm}^{-1}$ corresponds to $5 \pm 3$ [w/w\%], which is close to the critical

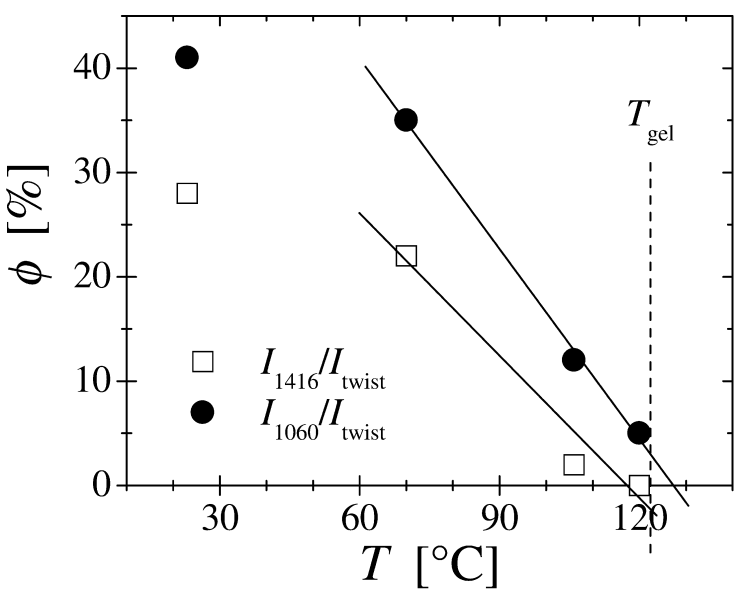

Fig. 9. Temperature dependence of Raman Crystallinity during partial melting of M-LLDPE as calculated from the intensity ratio of the $1416 \mathrm{~cm}^{-1}$ band to the overall intensity of the $\mathrm{CH}_{2}$ twisting signal (full squares) and from the intensity ratio for the $1060 \mathrm{~cm}^{-1}$ band (open circles).
Table 4

Area of Raman bands obtained from band deconvolution

\begin{tabular}{lllll}
\hline Peak $\left(\mathrm{cm}^{-1}\right)$ & $22{ }^{\circ} \mathrm{C}$ & $65-70{ }^{\circ} \mathrm{C}$ & $106-11{ }^{\circ} \mathrm{C}$ & $120{ }^{\circ} \mathrm{C}$ \\
\hline $1340-1241$ & 1.576 & 1.551 & 1.821 & 1.819 \\
1418 & 0.219 & 0.167 & 0.017 & 0.000 \\
1439 & 1.227 & 0.084 & 0.953 & - \\
1459 & 1.296 & 1.287 & 1.484 & - \\
1170 & 0.082 & 0.055 & 0.011 & 0.000 \\
1129 & 0.477 & 0.414 & 0.127 & 0.026 \\
1084 & 0.288 & 0.389 & 0.730 & 0.858 \\
1062 & 0.514 & 0.436 & 0.176 & 0.080 \\
\hline
\end{tabular}

crystallinity detected by DSC using the isothermal and stepcrystallization methods.

Because the vibration at $1060 \mathrm{~cm}^{-1}$ is sensitive to any long trans conformation, the difference observed using the two Raman bands may be attributed to the existence of crystal structures other than orthorhombic ones or to long trans sequences that have not crystallized. In fact, LLDPEs have been shown to contain appreciable amounts of a monoclinic phase [18]. For samples observed at higher temperatures, another explanation may be that thermal expansion increases the interchain distance in the orthorhombic unit cell to the degree that crystalline field splitting is eliminated.

\section{Conclusions}

Differences in molecular architecture of LLDPE have tremendous consequences for the rheological parameters during quiescent crystallization. The distribution of crystallizable sequence lengths is found to have profound influences on the viscoelastic properties of LLDPEs during crystallization and melting. Non-isothermal crystallization by slow cooling gives a narrower solidification interval for the highly heterogeneous copolymer ZN-LLDPE than for homogeneous M-LLDPE. This is attributed to a significant content of almost linear chains in $\mathrm{ZN}$ copolymer. The relationship between the widths of solidification interval and content of linear and/or low-branched chains was observed in experiments with blends of M-LLDPEs with HDPEs.

Crystallinity and local chain conformations in slightly crystallized M-LLDPE near the solid - melt transition were determined by Raman spectroscopy. Raman data suggest that a relatively small amount of crystallites is sufficient for the transition from melt-like to solid-like rheological properties of M-LLDPE.

It should be noted that the Raman observations are valid for the structures prepared by the partial melting procedure which are not necessarily identical with structures formed during the isothermal or non-isothermal crystallization of LLDPEs, often encountered in practical applications. The crystallite and spherulite structures are indeed strongly path 
dependent. The step melting procedure's repeated isothermal annealing facilitates perfection of crystallites and should produce gels with more perfect crystals than the other two routes, still even in highly annealed systems the content of perfect crystallites at a gel point is negligibly small, indicating that a melt-solid transition in LLDPE may occur even in the absence of well-defined crystalline structures.

The ability to predict the solidification behavior of LLDPEs based on their composition can be extremely useful for the optimization of such industrial processes as film blowing and blow molding, where polymer processing includes handling of slightly crystallized polymers near the gel point. Another practically important application is hotmelt adhesives containing LLDPEs. Indeed, for melt adhesives the solidification range should be narrow enough to allow for a rapid 'setting'; yet if a solidification occurs at very low under cooling (too narrow a solidification interval) the adhesive layer will not be able to wet the surfaces, resulting in a weak bonding [42].

Understanding of the rheological behavior of LLDPEs during crystallization will help to manufacture copolymers and blends of copolymers able to deform and solidify in a desired way under the influence of stress and temperature fields during processing and application.

\section{Acknowledgements}

The financial support of the MRSEC program at the University of Massachusetts, Amherst (NSF DMR 9809365) and of CUMIRP is gratefully acknowledged.

\section{References}

[1] Hsieh ET, Randall JC. Macromolecules 1982;15:1402-6.

[2] Hsieh ET, Randall JC. Macromolecules 1982;15:353-60.

[3] Kimura K, Shigemura T, Yuasa S. J Appl Polym Sci 1984;29: $3161-70$.

[4] Kimura K, Yuasa S, Maru Y. Polymer 1984;25:441-6.

[5] Besanson S, Minick J, Moet A, Chum S, Hiltner A, Baer E. J Polym Sci Polym Phys 1996;34:1301-15.

[6] Mirabella FM. J Polym Sci Polym Phys 2001;39:2800-18.
[7] Mathot VBF. In: Chung TC, editor. New advances in polyolefins. New York: Plenum Press; 1993. p. 121.

[8] Mirabella FM, Ford EA. J Polym Sci B Polym Phys 1987;25:777-90.

[9] Kim MH, Phillips PJ, Lin JS. J Polym Sci B Polym Phys 2000;38: 154-70.

[10] Wagner J, Abu-Iqyas S, Mohar K, Phillips PJ. Polymer 1999;40: 4717-30.

[11] Kim MH, Phillips PJ. J Appl Polym Sci 1998;70:1893-905.

[12] Alamo RG, Domszy R, Mandelkern L. J Phys Chem 1984;88: 6587-95.

[13] Alamo RG, Mandelkern L. Thermochim Acta 1994;238:155-201.

[14] Flory PJ. Trans Faraday Soc 1955;51:848-57.

[15] Crist B, Howard PR. Macromolecules 1999;32:3057-67.

[16] Alizadeh A, Richardson JX, McCartney S, Marand H, Cheung YW, Chum S. Macromolecules 1999;32:6221-35.

[17] Gelfer M, Winter HH. Macromolecules 1999;32:8974-81.

[18] Horst RH, Winter HH. Macromolecules 2000;33:130-6.

[19] Boutahar K, Carrot C, Guillet J. Macromolecules 1998;31:1921-9.

[20] Pogodina NV, Winter HH. Macromolecules 1998;31:8164-72.

[21] Young RJ, Lovell PA. Introduction to polymers. New York: Chapman and Hall; 1991. p. 284

[22] Hess W, Vilgis TA, Winter HH. Macromolecules 1988;21:2536-42.

[23] Chambon F, Winter HH. Polym Bull 1985;13:499-503.

[24] Winter HH, Chambon F. J Rheol 1986;30:367-82.

[25] Chambon F, Winter HH. J Rheol 1987;31:683-97.

[26] Schwittay C, Mours M, Winter HH. Faraday Discuss 1995;101: 93-104.

[27] te Nijenhuis K, Winter HH. Macromolecules 1989;22:411-4.

[28] Rull F, Priesto AC, Casado JM, Sobron F, Edwards HGM. Raman Spectrosc 1993;24:545.

[29] Wasserman SH, Graessley WW. Polym Engng Sci 1996;36:852-61.

[30] Wasserman SH, Graessley WW. J Rheol 1992;36:543-72.

[31] Lambert WS, Phillips PJ. Polymer 1996;3:3585-91.

[32] Richardson MJ, Flory PJ, Jackson JB. Polymer 1963;4:221-36.

[33] Akpalu Y, Kielhorn L, Hsiao BS, Stein RS, Russell TP, van Egmond J, Muthukumar M. Macromolecules 1999;32:765-70.

[34] Mandelkern L, Glotin M, Benson R. Macromolecules 1981;14: 22-34.

[35] Djabourov M. Polym Int 1991;25:135-43.

[36] Horst RH, Winter HH. Macromolecules 2000;33:7538-43.

[37] Strobl GR, Hagedorn W. J Polym Sci Polym Phys 1978;16:1181-93.

[38] Mutter R, Stille W, Strobl G. J Polym Sci Polym Phys 1993;31: 99-105.

[39] Mandelkern L, Alamo RG. Macromolecules 1995;28:2988-9.

[40] Rodriguez-Cabello JC, Martin-Monge J, Lagaron JM, Pastor JM. Macromol Chem Phys 1998;199:2767-76.

[41] Naylor CC, Meier RJ, Kip BJ, Williams KPJ, Mason SM, Conroy N, Gerrard DL. Macromolecules 1995;28:2969-78.

[42] Kinloch AJ. Adhesion and adhesives: science and technology. London: Chapman and Hall; 1987. (Chapter 5). 